\title{
Impacto de la intervención pedagógica en la competencia de cuidado
}

\section{Impact of Pedagogical Intervention on Care Competence}

\section{Impacto da intervenção pedagógica na competência do cuidado}

Roosby Gallardo Solarte, MSc ${ }^{1 *}$

Vilma Ortiz Nievas, $\mathrm{MSc}^{1}$

Recibido: 1. . de febrero de 2019 • Aceptado: 26 de febrero de 2021

Doi: https://doi.org/10.12804/revistas.urosario.edu.co/revsalud/a.10282

Para citar este artículo: Gallardo Solarte R, Ortiz Nievas V. Impacto de la intervención pedagógica en la competencia de cuidado. Rev Cienc Salud. 2021;19(2):1-15. https://doi.org/10.12804/revistas.urosario.edu.co/revsalud/a.10282

\section{Resumen}

Introducción: la enfermedad crónica es una problemática que ha ido en aumento año tras año, y con ello la aparición del cuidador familiar. Esta investigación procuró abordar el fenómeno de la cronicidad, con el fin de minimizar las complicaciones. El objetivo fue determinar el efecto de una propuesta educativa en dos instituciones de salud en Pasto (Colombia), sobre la competencia que debe tener el cuidador. Materiales y métodos: estudio experimental, aleatorizado, con la finalidad de probar el efecto que tiene la intervención del plan de egreso hospitalario a un grupo de 126 pacientes con hipertensión arterial y diabetes mellitus tipo 2 (grupo experimental), frente a otro grupo de pacientes con las mismas patologías, quienes recibieron intervención convencional de las instituciones involucradas (grupo control), para comparar los resultados entre estos dos grupos. Las pruebas estadísticas aplicadas fueron chi cuadrado y prueba exacta de Fisher para asegurar que fueran significativas. Resultados: los cuidadores familiares, en un $71 \%$, fueron de sexo femenino; el $38 \%$ estaba entre los 30 y los 44 años, y la competencia de cuidado mejoró en nueve de cada diez cuidadores evaluados. Esta proporción de incidencia fue mayor en las personas intervenidas con la estrategia pedagógica. Conclusión: la intervención pedagógica evidenció la importancia del componente educativo para generar cambios favorables en la población objeto de estudio que contribuyen a mejorar la competencia del cuidado del cuidador.

Palabras clave: enfermedad crónica; educación; cuidadores.

1 Universidad Mariana (Colombia). 
Introduction: Chronic disease is a recurring issue that has been increasing over years in parallel with the appearance of the family caregiver. The present research attempted to address the chronicity phenomenon in order to minimize complications. The objective of the present study was to determine the effect of an educational proposal in two health institutions located in Pasto based on the required care competence of the caregiver. Materials and methods: An experimental, randomized study was conducted to test the effect of the hospital discharge plan intervention on a group of 126 patients with arterial hypertension (Ант) and diabetes mellitus type 2 (experimental group) when compared to another group of patients with same pathologies, who received conventional intervention from the involved institutions (control group) in order to compare the results between the two groups. The chi square and Fisher's exact tests were used in order to ensure statistically significant results. Results: Among family caregivers, $71 \%$ were female; $38 \%$ were of age 30-44 years; and care competence improved in nine out of ten evaluated caregivers. The incidence rate was higher in people who underwent the pedagogical strategy. Conclusion: The pedagogical intervention revealed the importance of the educational component for generating favorable changes in the population under study, which in turn contributed to the improvement in the care competence of caregivers.

Keywords: Chronic disease; education; caregivers.

\section{Resumo}

Introdução: a doença crônica é uma problemática que vem aumentando ano após ano e, com ela, o surgimento do cuidador familiar. Esta pesquisa buscou abordar o fenômeno da cronicidade, a fim de minimizar suas complicações. O objetivo é verificar o efeito de uma proposta educacional em duas instituições de saúde de Pasto, sobre a competência que o cuidador deve ter. Materiais e métodos: estudo experimental, randomizado, com o objetivo de testar o efeito da intervenção do plano de alta hospitalar em um grupo de 126 pacientes com hipertensão arterial (нтА) e diabetes mellitus do tipo 2 (grupo experimental), em comparação com outro grupo de pacientes com as mesmas patologias, que receberam intervenção convencional das instituições envolvidas (grupo controle), para comparar os resultados entre os dois grupos. O teste estatístico aplicado foi o qui-quadrado e o teste de Fisher para testar a significância das diferenças. Resultados: $71 \%$ dos cuidadores familiares eram do sexo feminino; 38\% tinham entre 30 e 44 anos; a competência para cuidar melhorou em nove entre dez cuidadores avaliados; essa taxa de incidência foi maior nas pessoas tratadas com a estratégia pedagógica. Conclusão: a intervenção pedagógica possibilitou evidenciar a importância do componente educacional, gerando mudanças favoráveis na população em estudo, que contribuem para a melhoria da competência em cuidado desses cuidadores.

Palavras-chave: doença crônica; educação; cuidadores.

\section{Introducción}

a enfermedad crónica es un problema mundial que afecta de manera especial a los países las enfermedades crónicas, y las proyecciones epidemiológicas para las próximas décadas indican que la mayor parte de las patologías crónicas acrecentarán su prevalencia (2). La Organización Mundial de la Salud ha llamado a la comunidad internacional a incrementar sus esfuerzos y a fijar prioridades en la prevención y el combate a los padecimientos no 
transmisibles, que son considerados un importante reto para el desarrollo sostenible (3). Por otro lado, estos padecimientos son un desafío importante que afrontan los sistemas de salud en el mundo, asumido por el cambio poblacional, pues ha aumentado de manera significativa la edad adulta mayor y, por ende, sus alteraciones en salud (4), y Colombia no es una excepción.

Al respecto, es marcado el incremento de la expectativa de vida y de la prevalencia de enfermedades crónicas no trasmisibles, que, junto con la aparición de pluripatologías en el mismo sujeto, produce necesidades de salud cada más complicadas (5). Esta problemática se ha ido acrecentando año tras año y, con ella, la aparición del cuidador familiar, quien al estar atendiendo a su ser querido, requiere ser capacitado para ello, con el fin de contribuir al restablecimiento y a evitar reingresos hospitalarios de la persona enferma. El cuidado del paciente crónico, a partir de su diagnóstico, recae en el cuidador (en este caso familiar), quien acompaña y gestiona en la consulta médica y quien continúa con los tratamientos en el domicilio, apropiándose del rol de cuidado de manera permanente o brindando tiempo y esfuerzo al cuidado durante el tiempo que dure enfermedad (6).

Las enfermedades crónicas no transmisibles son un problema nacional de salud pública que repercute mayoritariamente en las poblaciones con debilidad económica y social. El costo incluye la evaluación de las consecuencias adversas reales que se presenta en los cuidadores (7). En este sentido, se considera fundamental incrementar el componente educativo en este tipo de poblaciones, ya que las enfermedades crónicas no transmisibles son prevenibles y, en su mayoría, estas se desencadenan por falta de estilos de vida saludables, como son la alimentación inadecuada, el sedentarismo, el consumo de tabaco y alcohol. Por otra parte, el cuidador será el que asuma las responsabilidades de cuidado de su familiar y quien evidenciará cambios importantes relacionados con mejores estilos de vida congruentes con lo social, lo laboral, lo personal, con los efectos negativos que tienen que ver con la sobrecarga y las pocas habilidades para ofrecer cuidado. Por lo anterior, es de relevancia la investigación, ya que pretendió medir la importancia del componente educativo dirigido a los cuidadores familiares, para mejorar la competencia de cuidado desde su hogar.

\section{Materiales y métodos}

— studio experimental con muestreo aleatorio estratificado según la enfermedad de base, Lperiodo de la enfermedad y tipo de complicación en órgano blanco. Como técnica de enmascaramiento se utilizó un simple ciego, es decir, los participantes desconocían el grupo de tratamiento que iban a recibir; en este caso, los que recibirían la intervención dada por los investigadores o la recibida por la institución de salud. Este estudio tuvo la finalidad de probar el efecto que tiene la intervención del plan de egreso hospitalario en un grupo 
de pacientes con hipertensión arterial y diabetes mellitus tipo 2 (grupo experimental) frente a otro con las mismas patologías, quienes recibieron la intervención convencional de las instituciones involucradas (grupo control), con el objetivo de comparar los resultados entre estos dos grupos, con la característica de haber estado hospitalizados entre enero y marzo de 2016, por complicaciones en órgano blanco. No ocurrieron pérdidas en el seguimiento.

La muestra fue de 126 pacientes, con su respectivo cuidador familiar (para un total de 252), de los cuales 63 fueron parte del grupo experimental y los 63 restantes fueron parte del grupo control, cada uno con sus respectivos cuidadores. La muestra fue calculada con un error estándar del $6 \%$ y una prevalencia $(p)$ del $22 \%$, correspondiente a hipertensión (hipertensión Pasto) (8), por brindar el mayor tamaño muestral; un índice de confianza del $95 \%$ y un aumento del $10 \%$, por deserción en el estudio. La prueba estadística aplicada para medir la competencia de cuidado fue chi cuadrado $\left(\chi^{2}\right)$, y para lograr asegurar que fuera significativa, la prueba exacta de Fisher.

\section{Plan de análisis}

La información recolectada en el trabajo de campo se digitalizó en el software Microsoft Excel $2010 ®$, en una plantilla prevalidada por medio de la función “validación de datos”, a efectos de evaluar la calidad de la digitación de una de cada cinco encuestas, por medio de la concordancia de los instrumentos físicos con lo digital, una vez consolidadas las bases de datos de cada uno de los dos instrumentos: encuesta de caracterización para el cuidado de una díada persona con enfermedad crónica-cuidador familiar (9) y Competencia para el cuidado en el hogar (GCPC-UNCPC), versión abreviada con las seis dimensiones: 1) nivel de conocimiento, 2) nivel de unicidad, 3) competencia en los procesos instrumentales y procedimentales del cuidador, 4) bienestar, 5) anticipación y manejo de riesgo social y 6) interacción del cuidador con el paciente y la familia (10). Estos instrumentos los proporcionó el Grupo de Cuidado de Enfermería al Paciente Crónico de la Facultad de Enfermería de la Universidad Nacional de Colombia. Con autorización de uso, se procedió al análisis por medio de tablas dinámicas de Microsoft Excel $2010 \circledast$ y de análisis bivariados con el software spss, versión 22.

En el análisis univariado de cada uno de los ítems de la díada se evaluó la composición sociodemográfica y clínica de cuidadores y de pacientes, mediante tablas con las frecuencias absolutas y relativas; además, se evaluó el grado de competencia pre y pos del cuidador familiar de los pacientes crónicos tanto del grupo experimental como del grupo control. Posteriormente, se creó una variable que indicaba si entre la medición pre y pos había una mejoría en la competencia del cuidador ( 1 = mejoró; 0 = no mejoró), entre el grupo control y el experimental, segmentando los análisis por grupo de edad, sexo y nivel educativo; así como analizando la diferencia y la razón de riesgo de la mejoría en la competencia de los cuidadores familiares. 
En el grupo experimental, el tiempo de participación en el estudio fue dado en tres momentos: 1) una medición inicial, en la cual se exploraron las competencias de cuidado de los cuidadores hacia el paciente usando una encuesta que permitió evaluar las variables en mención. 2) Una intervención educativa que tuvo en cuenta las seis dimensiones de cuidado, utilizando una cartilla educativa impresa, denominada ¿Convives con hipertensión arterial o diabetes, y quieres saber su cuidado?, donada a los cuidadores de pacientes intervenidos. Esta actividad la llevó a cabo el profesional de enfermería con una duración de 30 minutos, en dos ocasiones, para brindar educación e instrucción al paciente y al cuidador familiar durante la estancia hospitalaria y durante el egreso en temas relacionados con hipertensión arterial, diabetes, obesidad, factores de riesgo, manejo de medicamentos, importancia del apoyo social-espiritual y desarrollo de habilidades. 3) La medición final, que se dio en los dos grupos, tanto en el experimental como en el control. En este último fue en dos momentos: uno en la medición inicial y otro en la medición final, cuya intervención fue la que la institución prestadora de servicios ejecuta. Se utilizaron dos instrumentos: el de caracterización del cuidador familiar del Grupo de Cuidado al Paciente Crónico y su Familia, de la Universidad Nacional de Colombia, y la capacidad del cuidador para el cuidado, adaptado por el Grupo de Cuidado al Paciente Crónico y su Familia, de la Facultad de Enfermería de la Universidad Nacional de Colombia.

Los autores autorizaron el uso de los instrumentos, previa validación. El estudio y el artículo se derivan de la investigación Programa para la disminución de la carga de la enfermedad crónica en Colombia. El proyecto tuvo en cuenta las normas éticas emanadas de la Declaración de Helsinki y de la Resolución 8430 de 1993 del Ministerio de Salud de Colombia $(11,12)$. Se respetó la confidencialidad y el anonimato de los participantes. También se obtuvo el aval del Comité de Ética de la institución (según el Acta 001 del Comité de Investigación) y se diligenció el consentimiento informado.

\section{Resultados}

e los 126 cuidadores familiares captados, el $71 \%$ perteneció al género femenino, el $38 \%$ de
los cuidadores se encontraba dentro del rango de edad de 30 a 44 años, el lugar de residencia fue el urbano (representado en un 83\%), un $62 \%$ estaba casado o se hallaba en unión libre, un $32 \%$ se dedicaba al hogar y en escolaridad predominó la primaria, con un 37\% (tabla 1). 
Tabla 1. Características sociodemográficas de los cuidadores de pacientes crónicos

\begin{tabular}{|c|c|c|c|}
\hline \multirow{2}{*}{\multicolumn{2}{|c|}{ Características clínicas y sociodemográficas }} & \multicolumn{2}{|c|}{ Cuidadores } \\
\hline & & \multirow{2}{*}{$\begin{array}{c}\mathbf{n} \\
38\end{array}$} & \multirow{2}{*}{$\begin{array}{c}\mathbf{\%} \\
30.2\end{array}$} \\
\hline \multirow{2}{*}{ Institución } & Institución 1 & & \\
\hline & Institución 2 & 88 & 69.8 \\
\hline \multirow{2}{*}{ Género } & Femenino & 90 & 71.4 \\
\hline & Masculino & 36 & 28.6 \\
\hline \multirow{5}{*}{ Grupo de edad } & 18 a 29 & 16 & 12.7 \\
\hline & 30 a 44 & 48 & 38.1 \\
\hline & 45 a 54 & 27 & 21.4 \\
\hline & 55 a 64 & 20 & 15.9 \\
\hline & 65 y más & 15 & 11.9 \\
\hline \multirow{3}{*}{ Estado civil } & Casado-unión libre & 78 & 61.9 \\
\hline & Separado-viudo & 39 & 31.0 \\
\hline & Soltero(a) & 9 & 7.1 \\
\hline \multirow{5}{*}{ Ocupación } & Empleado(a) & 27 & 21.4 \\
\hline & Estudiante & 3 & 2.4 \\
\hline & Hogar & 40 & 31.7 \\
\hline & Otros & 22 & 17.5 \\
\hline & Independiente & 34 & 27.0 \\
\hline \multirow{5}{*}{ Nivel de escolaridad } & Ninguno & 3 & 2.4 \\
\hline & Primaria & 47 & 37.3 \\
\hline & Bachillerato & 45 & 35.7 \\
\hline & Técnico & 10 & 7.9 \\
\hline & Universitario & 21 & 16.7 \\
\hline \multicolumn{2}{|l|}{ Total } & 126 & 100 \\
\hline
\end{tabular}

La evaluación de impacto de la intervención pedagógica sobre la competencia del cuidado familiar mejoró en nueve de cada diez cuidadores, lo que se ve representado en un $91 \%$. Esta proporción de incidencia fue mayor en las personas intervenidas con la estrategia pedagógica: ¿Convives con hipertensión arterial o diabetes, y quieres saber su cuidado? (95 \%), frente a las personas que recibieron las indicaciones propias del plan de egreso hospitalario de la institución, en la que se captó al $86 \%$. Al evaluar por medio de la prueba $\chi^{2}$ si existe relación entre el tipo de intervención y la mejoría en las competencias del cuidador, se rechazó la hipótesis nula (Ho: no hay relación entre el tipo de intervención y la mejoría en la competencia del cuidador); sin embargo, con frecuencias menores a 5 en la tabla de $2 \times 2$ con el estadístico ajustado y la prueba exacta de Fisher no se rechazó la hipótesis nula (Ho: 
no hay relación entre el tipo de intervención y la mejoría en la competencia del cuidador). $\mathrm{Al}$ analizar la razón de riesgo, hay un 10\% más de probabilidad de mejorar las competencias del cuidador en la población intervenida frente a la no intervenida; empero, el intervalo de confianza al $95 \%$ indica que no es significativo por contener el uno, y la diferencia de riesgos fue del 9,5\% (tabla 2).

Tabla 2. Impacto de la intervención educativa en la competencia del cuidador

\begin{tabular}{|c|c|c|c|c|c|}
\hline \multirow{3}{*}{ Intervención } & \multicolumn{4}{|c|}{ Competencia del cuidador(a) } & \multirow{3}{*}{ Total general } \\
\hline & \multicolumn{2}{|c|}{ Mejoró } & \multicolumn{2}{|c|}{ No mejoró } & \\
\hline & $\mathbf{n}$ & $\%$ & $\mathbf{n}$ & $\%$ & \\
\hline Intervención & 60 & 95.2 & 3 & 4.8 & 63 \\
\hline Control & 54 & 85.7 & 9 & 14.3 & 63 \\
\hline Total general & 114 & 90.5 & 12 & 9.5 & 126 \\
\hline \multicolumn{3}{|c|}{ Prueba } & Valor & \multicolumn{2}{|c|}{ Valor de $p$ (1-cola) } \\
\hline \multicolumn{3}{|c|}{$\chi^{2} \sin$ corrección } & 3.316 & \multicolumn{2}{|c|}{0.03431} \\
\hline \multicolumn{3}{|c|}{ Exacto de Fisher } & & \multicolumn{2}{|c|}{0.06322} \\
\hline \multirow{2}{*}{\multicolumn{3}{|c|}{ Indicadores epidemiológicos }} & \multirow{2}{*}{ Valor } & \multicolumn{2}{|c|}{ Intervalo de confianza $95 \%$} \\
\hline & & & & Inferior & Superior \\
\hline \multicolumn{3}{|c|}{ Riesgo en expuestos (\%) } & 95.2 & 86.4 & 98.9 \\
\hline \multicolumn{3}{|c|}{ Riesgo en no expuestos (\%) } & 85.7 & 74.8 & 92.5 \\
\hline \multicolumn{3}{|l|}{ Riesgo total (\%) } & 90.5 & 83.96 & 94.6 \\
\hline \multicolumn{3}{|l|}{ Razón de riesgo } & 1.1 & 0.99 & 1.246 \\
\hline \multicolumn{3}{|c|}{ Diferencia de riesgo (\%) } & 9.5 & -0.6 & 19.6 \\
\hline
\end{tabular}

Para la competencia del cuidador se tuvo en cuenta el instrumento Competencia para el cuidado en el hogar, con las seis dimensiones. Entre las mujeres, la competencia en el cuidado de pacientes crónicos mejoró en nueve de cada diez cuidadoras evaluadas (90\%). Esta proporción de incidencia fue mayor en las mujeres intervenidas con la estrategia pedagógica (96\%), frente a aquellas que recibieron las indicaciones propias del plan de egreso hospitalario de la institución, en la que se captó a un $84 \%$. Al evaluar por medio de la prueba $\chi^{2}$, sí existe relación entre el tipo de intervención y la mejoría en las competencias de las cuidadoras. Se seleccionaron frecuencias menores a 5 en la tabla de $2 \times 2$ con el estadístico ajustado y la prueba exacta de Fisher, con lo cual no se rechazó la hipótesis nula. La razón de riesgo indicó que hay un $13 \%$ más de probabilidad de mejorar las competencias de las cuidadoras intervenidas frente a las no intervenidas (tabla 3 ). 
Tabla 3. Impacto de la intervención educativa en la competencia de las mujeres cuidadoras

\begin{tabular}{|c|c|c|c|c|c|}
\hline \multirow{3}{*}{ Intervención } & \multicolumn{4}{|c|}{ Competencia del cuidador(a) } & \multirow{3}{*}{ Total general } \\
\hline & \multicolumn{2}{|c|}{ Mejoró } & \multicolumn{2}{|c|}{ No mejoró } & \\
\hline & $\mathbf{n}$ & $\%$ & $\mathbf{n}$ & $\%$ & \\
\hline Expuestos & 43 & 95.6 & 2 & 4.4 & 45 \\
\hline No expuestos & 38 & 84.4 & 7 & 15.6 & 45 \\
\hline Total general & 81 & 90.0 & 9 & 10.0 & 90 \\
\hline \multicolumn{3}{|c|}{ Prueba } & Valor & \multicolumn{2}{|c|}{ Valor de $p$ (1-cola) } \\
\hline \multicolumn{3}{|l|}{$\chi^{2} \sin$ corrección } & \multirow[t]{2}{*}{3.086} & \multicolumn{2}{|c|}{0.03948} \\
\hline \multicolumn{3}{|l|}{ Exacto de Fisher } & & \multicolumn{2}{|c|}{0.07860} \\
\hline \multirow{2}{*}{\multicolumn{3}{|c|}{ Indicadores epidemiológicos }} & \multirow{2}{*}{ Valor (\%) } & \multicolumn{2}{|c|}{ Intervalo de confianza $95 \%$} \\
\hline & & & & Inferior & Superior \\
\hline \multicolumn{3}{|l|}{ Riesgo en expuestos (\%) } & 95.6 & 84.4 & 99.6 \\
\hline \multicolumn{3}{|c|}{ Riesgo en no expuestos (\%) } & 84.4 & 70.9 & 92.6 \\
\hline \multicolumn{3}{|l|}{ Riesgo total (\%) } & 90 & 81.88 & 94.8 \\
\hline \multicolumn{3}{|l|}{ Razón de riesgo } & 1.132 & 0.983 & 1.302 \\
\hline \multicolumn{3}{|c|}{ Diferencia de riesgo (\%) } & 11.1 & -1.07 & 23.3 \\
\hline
\end{tabular}

Entre los hombres, la competencia en el cuidado de pacientes crónicos mejoró en nueve de cada diez cuidadores evaluados (92\%). Esta proporción de incidencia fue mayor en los hombres intervenidos con la estrategia pedagógica (94\%) frente a los que recibieron las indicaciones propias del plan de egreso hospitalario de la institución, en la que se captó a un $90 \%$. Al evaluar por medio de la prueba $\chi^{2}$, la cual dio 3.086, nos permite determinar que sí existe relación entre el tipo de intervención y la mejoría en las competencias del cuidado de los cuidadores (con un valor de $p$ menor de 0.039), que apoya la asociación entre las variables. La razón de riesgo que hay es de un $6 \%$ más de probabilidad de mejorar las competencias de los cuidadores intervenidos, frente a los no intervenidos (tabla 4). 
Tabla 4. Impacto de la intervención educativa en la competencia de los hombres cuidadores

\begin{tabular}{|c|c|c|c|c|c|}
\hline \multirow{3}{*}{ Intervención } & \multicolumn{4}{|c|}{ Competencia del cuidador(a) } & \multirow{3}{*}{ Total general } \\
\hline & \multicolumn{2}{|c|}{ Mejoró } & \multicolumn{2}{|c|}{ No mejoró } & \\
\hline & $\mathbf{n}$ & $\%$ & $\mathbf{n}$ & $\%$ & \\
\hline Expuestos & 17 & 94.4 & 1 & 5.6 & 18 \\
\hline No expuestos & 16 & 88.9 & 2 & 11.1 & 18 \\
\hline Total general & 33 & 91.7 & 3 & 8.3 & 36 \\
\hline \multicolumn{3}{|c|}{ Prueba } & Valor & \multicolumn{2}{|c|}{ Valor de $p$ (1-cola) } \\
\hline \multicolumn{3}{|c|}{$\chi^{2} \sin$ corrección } & \multirow[t]{2}{*}{0.3636} & \multicolumn{2}{|c|}{0.2732} \\
\hline \multicolumn{3}{|c|}{ Exacto de Fisher } & & \multicolumn{2}{|c|}{0.5000} \\
\hline \multirow{2}{*}{\multicolumn{3}{|c|}{ Indicadores epidemiológicos }} & \multirow{2}{*}{ Valor } & \multicolumn{2}{|c|}{ Intervalo de confianza $95 \%$} \\
\hline & & & & Inferior & Superior \\
\hline \multicolumn{3}{|c|}{ Riesgo en expuestos (\%) } & 94.4 & 72.4 & 100 \\
\hline \multicolumn{3}{|c|}{ Riesgo en no expuestos (\%) } & 88.9 & 65.9 & 98.1 \\
\hline \multicolumn{3}{|l|}{ Riesgo total (\%) } & 91.7 & 77.4 & 97.9 \\
\hline \multicolumn{3}{|l|}{ Razón de riesgo } & 1.063 & 0.8716 & 1.295 \\
\hline \multicolumn{3}{|c|}{ Diferencia de riesgo (\%) } & 5.6 & -12.4 & 23.5 \\
\hline
\end{tabular}

\section{Discusión}

$\mathrm{L}$

as características sociodemográficas de las personas cuidadoras son similares en diversos estudios. Una investigación en cinco regiones de Colombia describe que el 74.7\% de los cuidadores familiares son mujeres, el 39.3\% tiene menos de 35 años, el 43.7\% está entre los 36 y los 59 años y el 16.9\% tiene 60 años o más (13). En otro estudio realizado en Villavicencio con cuidadores de pacientes crónicos se encontró que el $78.89 \%$ fueron de género femenino y con edades entre los 31 y los 70 años, que viven en los estratos 1 (25\%) y 2 (47\%), y para quienes dichas condiciones restringen el acceso a servicios de salud. Estos datos son similares a los hallados en esta investigación (14). Cerquera et al. reportan semejanza en el género, ya que el femenino es el de más alto porcentaje (15). Otra investigación con cuidadores de pacientes crónicos evidenció que los cuidadores, en un $43.7 \%$, están entre los 36 y los 59 años de edad (16). Entre tanto, en un estudio llevado a cabo en Cartagena, el $51 \%$ está en edades menores a los 35 años, datos contrarios a esta investigación (17). Respecto al estado civil, las autoras afirman que, en su gran mayoría, los cuidadores están casados o viven en unión libre, se dedican a actividades del hogar o realizan trabajos formales y pertenecen al estrato 1, datos que coinciden con el presente estudio $(18,19)$. 
Relativo a la escolaridad, otra investigación mostró que los cuidadores, en mayor cantidad, lograron el nivel técnico, seguido del bachillerato (20). En las regiones Andina y Caribe se encuentran más cuidadores con bachillerato completo, y en las regiones de la Orinoquía y el Pacífico, más personas con primaria incompleta; en la región de la Amazonía, los cuidadores presentaron haber cursado hasta un nivel técnico, información que coincide con esta investigación.

La habilidad de cuidado se ha evaluado en Latinoamérica y en diferentes regiones de Colombia, y se ha hallado que es poca en la población de cuidadores (21). En este orden de ideas, se ha demostrado que es directamente proporcional la habilidad de cuidado con la competencia de cuidado. Respecto a la competencia del cuidador, la atención informal se sitúa en el centro del debate sobre las políticas de bienestar en la actualidad, debido al creciente aumento de la demanda de cuidados y disponibilidad de cuidadores informales (22). Por otra parte, las familias, por ser el núcleo de la sociedad, tienen un alto grado de responsabilidad sobre sus familiares enfermos, y los cuidadores no están preparados para su atención y asistencia, especialmente de aquellos con una enfermedad crónica.

Referente a este tema, estudios reportan que la presencia de un miembro de la familia enfermo requiere cuidados y genera una nueva dinámica familiar, situación que puede provocar importantes cambios dentro de la estructura familiar y los correspondientes roles que a partir de ahora tendrán que asumir (23). En esta investigación, la evaluación del impacto de la intervención pedagógica sobre la competencia del cuidado familiar mejoró en nueve de cada diez cuidadores; mientras que otros trabajos, relacionados con el desarrollo de esta competencia, referenciaron haber encontrado un apoyo social con las tecnologías de la información y comunicación, como método práctico y viable para promover la atención a los cuidadores de personas que padecen de enfermedades crónicas (24). Por otra parte, otro estudio realizado en Cúcuta con pacientes oncológicos permitió evidenciar que el grado de competencia de los cuidadores familiares principales de las personas que se encuentran en tratamiento de quimioterapia era calificado como alto por el $82.1 \%$ (101) de los encuestados, y como de mediano nivel de competencia, por el 17.9\% (22), actuaciones que deducen seguir con recomendaciones dadas por el equipo de salud (25).

En cuanto a la eficacia que tienen las intervenciones educativas con cuidadores, un estudio realizado en Perú documentó que una intervención con videos educativos logró mejorar significativamente tanto las prácticas como el conocimiento respecto al manejo del accidente cerebrovascular subagudo (26). Este trabajo halló un incremento del 50.74\% del nivel de práctica de los cuidadores de pacientes crónicos. De igual manera, otro estudio experimental, denominado "Eficacia de una intervención educativa individual en el dolor lumbar de cuidadores no profesionales de personas dependientes Programa TRANSFE”, demostró que las 
intervenciones educativas son estadísticamente significativas para todas las variables estudiadas en esta investigación en el grupo experimental; no así en el grupo control, donde solo se mejoran los índices de apoyo social percibido (27).

Al respecto de la eficacia del Programa transfe (a los tres meses) en relación con la formación estándar, la intervención educativa propuesta consigue resultados estadísticamente significativos. La individualizada es efectiva sobre el dolor lumbar que padecen los cuidadores de personas dependientes, con respecto a sus tres dimensiones estudiadas: presencia, nivel e incapacidad, así como en términos de sobrecarga, apoyo social percibido y calidad de vida relacionada con la salud. Así mismo, la intervención educativa individualizada es más efectiva que la estándar en relación con el dolor lumbar. Estos resultados encontrados en estas investigaciones permiten, una vez más, evidenciar cómo las intervenciones educativas con diferentes estrategias contribuyen a mejorar la competencia del cuidado del cuidador.

Por otra parte, en un estudio llevado a cabo en Bogotá se hizo referencia sobre la importancia que reviste el diseñar e implementar intervenciones en salud con el objetivo de aportar al mejoramiento de la calidad de esta, ante la necesidad de gestionar la salud desde lo preventivo y lo educativo, más aun teniendo en cuenta que las guías y protocolos dirigidos al diagnóstico y manejo clínico de las enfermedades crónicas no contemplan al paciente crónico en estado de total dependencia y necesidad de cuidado totalmente compensatorio. Ello debería permitir el diseño e implementación de intervenciones integrales, que son de apoyo a los cuidadores, a fin de favorecer el cuidado de su familiar (28).

En este mismo sentido, otra investigación evidenció la importancia de desarrollar estrategias participativas que repercutan en el empoderamiento de las habilidades del cuidado, que fomenten la adherencia a los cuidados y eviten complicaciones y que logren mejores desenlaces en salud, más aún considerando que la población es cada vez más adulta y vulnerable. De ahí que sea fundamental mejorar el nivel educativo de la díada para fortalecer sus conocimientos y llevarlos a asumir cambios en el comportamiento y lograr conductas permanentes que mantengan su grado de funcionalidad, actividad mental y autocuidado; además, apoyo psicosocial que impacte de manera positiva su calidad de vida (29).

De esta manera, se puede deducir que toda intervención educativa desde las diferentes formas como se aborde, sin distingo de diversas metodologías, se convierte en una importante herramienta de apoyo a los cuidadores, ya que les permite mejorar sus competencias frente al cuidado del enfermo crónico. Tal situación se evidencia en los referentes teóricos encontrados y se confirma con los resultados de la presente investigación.

En conclusión, los cuidadores familiares son, en su mayoría, personas entre los 30 y los 54 años, de género femenino, con un nivel educativo básico, dedicadas a labores del hogar, de estratos 1 y 2 . La intervención pedagógica frente al plan de egreso hospitalario habitual evidenció una mejora significativa en la competencia del cuidado del cuidador, en mayor proporción en las mujeres intervenidas con la estrategia pedagógica (un 95.6\%) que en los 
hombres, cuyo rango estadístico fue del 94.4\%. En general, la competencia en el cuidado de pacientes crónicos mejoró en nueve de cada diez cuidadores evaluados (90.5\%). Esta proporción de incidencia fue mayor en las personas intervenidas con la estrategia pedagógica (95.2\%) frente a las personas que recibieron las indicaciones propias del plan de egreso hospitalario de la institución en la que se captó (85.7\%).

$\mathrm{Al}$ evaluar por medio de la prueba $\chi^{2}$, sí existe relación entre el tipo de intervención y la mejoría en las competencias el cuidador y se rechaza, en este sentido, la hipótesis nula (Ho: no hay relación entre el tipo de intervención y la mejoría en la competencia del cuidador). Al analizar la razón de riesgo, indica que hay un 10\% más de probabilidad de mejorar las competencias del cuidador en población intervenida frente a la no intervenida. En cuanto a este resultado, los investigadores consideran que pudo influir el tiempo que se empleó la aplicación de la intervención educativa o, por otro lado, el plan de egreso utilizado por la institución, que estuvo bien fundamentado, al abarcar aspectos considerados dentro de la cartilla educativa utilizada en la investigación. Sin embargo, los estudios respecto al componente educativo versus cuidador de paciente crónico revelan que se evidencian cambios en el comportamiento, porque toman con más responsabilidad la labor de cuidado.

Dentro de las fortalezas que tuvo el estudio, los cuidadores recibieron capacitación mediante una intervención educativa estructurada y validada por expertos frente a la labor de cuidado. La cartilla fue utilizada por el personal de salud como insumo importante para complementar la educación dada a los pacientes y cuidadores cuando obtienen el alta hospitalaria. También hubo capacitación a estudiantes encargados de aplicar la intervención del plan de egreso hospitalario. Este trabajo servirá de base para una mejora continua para las propuestas educativas dirigidas a pacientes con enfermedad crónica y cuidadores. Otra fortaleza fue el uso de instrumentos previamente validados, que le dan robustez al estudio.

Como limitantes se encontraron el espacio, en términos de tiempo entre la intervención y el seguimiento, que fue reducido, por cuanto los pacientes tenían una estancia corta dentro de la institución hospitalaria, y quienes egresaban en su gran mayoría no eran del municipio de Pasto, por lo que fue necesario evaluar los conocimientos posintervención en un lapso corto, a fin de para evidenciar los cambios dentro de la misma institución antes de que egresaran.

\section{Agradecimientos}

$A_{\text {tes: Natalia Lasso y Simón Giraldo. A la Clínica Nuestra Señora de Fátima y al Hospital }}^{\text {la Universidad Mariana, a la Secretaría de Salud Municipal de Pasto, con sus participan- }}$ Universitario Departamental de Nariño, por hacer posible la realización de la investigación. A la Universidad Nacional de Colombia, por las asesorías y por proporcionar los instrumentos utilizados en la investigación. 


\section{Financiación}

a financiación fue realizada por la Universidad Mariana y la Secretaría Municipal de
Salud de Pasto.

\section{Contribución de los autores}

a elaboración y contribuciones intelectuales sustantivas del artículo y la búsqueda de
referentes bibliográficos fue de las dos autoras, proporcional al trabajo realizado en la elaboración y ajustes del artículo.

\section{Conflicto de intereses}

Ninguno declarado.

\section{Referencias}

1. Barreto Osorio RV, Coral Ibarra RC, Campos MS, Gallardo Solarte RK, Ortiz Nievas VT. Cuidadores y cuidadoras familiares de personas con enfermedad crónica en Colombia: más similitudes que diferencias. Revista Científica Salud Uninorte. 2015;31(2):255-65. https://rcientificas.uninorte.edu.co/index.php/salud/article/view/6988

2. Mármol López MI, Miguel Montoya I, Montejano Lozoya R, Escribano Pérez A, Gea Caballero V, Hontangas Ruiz A. Impacto de las intervenciones enfermeras en la atención a la cronicidad en España: revisión sistemática. Rev Esp Salud Publica. 2018; 92:e201806032.

3. Tamayo-Verdecia A. Prevención de enfermedades crónicas no transmisibles: a propósito de una reflexión. Revista Finlay [internet]. 2019 [citado 2020 may 13];9(4). Disponible en: http://revfinlay.sld.cu/index.php/finlay/article/view/725

4. Oviedo Córdoba HR, Rivas Castro AC, Caballero Zambrano M. Sociodemografía y estrés en enfermeras cuidadoras de pacientes crónicos. Rev Cubana Enferm [internet]. 2019 [citado 6 abr 2020];34(4). Disponible en: http://revenfermeria.sld.cu/index.php/enf/article/view/1638

5. Hernández Zambrano SM, Carrillo Algarra AJ, Castiblanco Montañez RA, Chaparro Díaz OL, Carreño Moreno SP, Gonzalo Jiménez E. Componentes del modelo de gestión de casos en la atención de la persona en condición de pluripatología y sus cuidadores familiares en Colombia. Paraninfo Digital [internet]. 2019;13(29):1. Disponible en: http:// ciberindex.com/c/pd/e124 
6. Galvis-López CR, Aponte-Garzón LH, Pinzón-Rocha ML. Percepción de la calidad de vida de cuidadores de pacientes asistentes a un programa de crónicos, Villavicencio, Colombia. Aquichan. 2016;16(1):104-15. https://doi.org/10.5294/aqui.2016.16.1.11

7. Gallardo-Solarte K, Benavides-Acosta FP, Rosales-Jiménez R. Costos de la enfermedad crónica no transmisible: la realidad colombiana. Rev Cienc Salud. 2016;14(1):103-14. https://doi.org/10.12804/revsalud14.01.2016.09

8. Alcaldía Municipal de Pasto, Secretaría Municipal Pasto. Base de Datos Única de Afiliados (BDUA) [internet]. 2017. Disponible en: https://www.pasto.gov.co/index.php/nuestras-dependencias/secretaria-de-salud

9. Chaparro L, Sánchez B, Carrillo GM. Encuesta de caracterización del cuidado de la diada cuidador familiar - persona con enfermedad crónica. Rev Cienc Cuidad. 2014;11(2):31-45.

10. Carrillo GM, Sánchez Herrera B, Arias Rojas EM. Validación de un instrumento para la evaluación de la competencia de cuidado en el hogar del cuidador familiar de una persona con enfermedad crónica. Investig Educ Enferm. 2015;33(3):449-55. https://doi. org/10.17533/udea.iee.v33n3a08

11. Declaración de Helsinki de la Amm: principios éticos para las investigaciones médicas en seres humanos [internet]. [Citado 2018 mar 12]. Disponible en: https://www.wma.net/ es/policies-post/declaracion-de-helsinki-de-la-amm-principios-eticos-para-las-investigaciones-medicas-en-seres-humanos/

12. Resolución 8430/1993 del 4 de octubre, por la cual se establecen normas científicas, técnicas y administrativas para la investigación en salud [internet]. Ministerio de Salud [internet]. Disponible en: https://www.minsalud.gov.co/sites/rid/Lists/BibliotecaDigital/ RIDE/DE/DIJ/RESOLUCION-8430-DE-1993.PDF

13. Barrera-Ortiz L, Campos MS, Gallardo-Solarte K, Coral Ibarra RC, Hernández-Bustos A. Soporte social percibido por las personas con enfermedad crónica y sus cuidadores familiares en cinco macro regiones geográficas de Colombia. Rev Univ Salud. 2016;18(1):102-12.

14. Aponte-Garzón LH, Pinzón-Rocha ML, Galvis-López CR. Nivel de funcionalidad de los enfermos crónicos y su relacion con la calidad de vida de los cuidadores informales. Enferm Global. 2014;33:191-200.

15. Cerquera Córdoba AM, Matajira Camacho J, Pabón Poches DK. Caracterización de una muestra de cuidadores formales de pacientes con trastorno neurocognitivo mayor en Bucaramanga. Revista Virtual Universidad Católica del Norte. 2016;47:4-19.

16. Ballesteros JE, Rodríguez Quenza AM, Cantor Chávez M, Peñalosa González GC, Valcárcel DE. Caracterización y percepción de carga de cuidadores familiares de personas con enfermedad crónica en Arauca. Orinoquía 2015;19(1):100-5 https://doi. org/10.22579/20112629.343

17. Montalvo Prieto A, Flórez Torres IE. Características de los cuidadores de personas en situación de cronicidad. Cartagena (Colombia): un estudio comparativo. Salud Uninorte. 2008;24(2):181-90.

18. Herrera A, Flórez IE, Romero E, Montalvo A. Soporte social a cuidadores familiares de personas con enfermedad crónica en cartagena. Aquichan. 2012;12(3):286-97. 
19. Sánchez Herrera B, Carrillo González GM, Corredor LR. Caracterización y carga del cuidado en hombres cuidadores familiares de personas con enfermedad crónica en Colombia. Arch Med (Col). 2014;14(2):219-30.

20. García-Calvente MM, Mateo-Rodríguez I, Maroto-Navarro G. El impacto de cuidar, en la salud y la calidad de vida de las mujeres. Gac Sanit. 2004;18(5):83-92.

21. Torres-Pinto X, Carreño-Moreno S, Chaparro-Díaz L. Factores que influencian la habilidad y sobrecarga del cuidador familiar del enfermo crónico. Rev Univ Ind Santander Salud. 2017;49(2):330-8. https://doi.org/10.18273/revsal.v49n2-2017006

22. Blanco Martínez L, Simón Álvarez A, Sánchez Fernández A. Caracterización biopsicosocial de los cuidadores informales de pacientes con enfermedad crónica o terminal. Rev Cuba Enf. 2016;32(3).

23. López Gil MJ, Orueta Sánchez R, Gómez-Caro S, Sánchez Oropesa A, Carmona de la Morena J, Alonso Moreno FJ. El rol de cuidador de personas dependientes y sus repercusiones sobre su calidad de vida y salud. Rev Clín Med Fam. 2009;2(7):332-9.

24. Cárdenas-Corredor DC, Melenge-Díaz B, Pinilla J, Carrillo-González GM, Chaparro-Díaz L. Soporte social con el uso de las тic para cuidadores de personas con enfermedad crónica: un estado del arte. Aquichán. 2010;10(3):204-10. https://doi.org/10.5294/aqui.2010.10.3.2

25. Montalvo Prieto A, Fajardo Rocha H. Competencias del cuidador familiar para cuidar a su familiar que recibe quimioterapia. Rev Cienc Cuidado. 2019;16(2):95-107. https:// doi.org/10.22463/17949831.1612

26. Sánchez Huamash CM. Eficacia del uso de videos educativos para cuidadores de pacientes en fase subaguda del accidente cerebrovascular [tesis de maestría en internet]. Lima: Universidad Peruana Cayetano Heredia; 2019. Disponible en: http://repositorio. upch.edu.pe/bitstream/handle/upch/6574/Eficacia_SanchezHuamash_Claudia.pdf?sequence=1\&isAllowed $=\mathrm{y}$

27. Ortiz Mallasen V. Estudio experimental sobre la eficacia de una intervención educativa individual en el dolor lumbar de cuidadores no profesionales de personas dependientes Programa transfe [tesis doctoral]. Madrid: Universitat Jaume I; 2019. https://doi. org/10.6035/14103.2019.117377

28. Lozano-Zamora AM, Beltrán-Medina AM. Aspectos relevantes para el diseño de un programa de capacitación dirigido al cuidador primario de pacientes crónicos [trabajo de grado en internet]. Bogotá: Universidad del Rosario; 2019 [citado 2020 jul 14]. Disponible en: https://repository.urosario.edu.co/bitstream/handle/10336/20368/LozanoZamoraAnaMar\%EDa-2019.pdf;jsessionid=12E126343510BECAAB5B29FD0C6DE410?sequence=1

29. Cantillo-Medina CP, Ramírez-Perdomo CA, Perdomo-Romero AY. Habilidad de cuidado en cuidadores familiares de personas con enfermedad crónica y sobrecarga percibida. Cienc Enferm. 2018;24:16. https://doi.org/10.4067/s0717-95532018000100216 